$\$$ Research Square

\title{
Evaluation of Different Types of Face Masks To Limit The Spread of SARS-CoV-2 - A Modeling Study
}

Brian M. Gurbaxani ( $\sim$ buw8@cdc.gov )

Centers for Disease Control and Prevention

Andrew N. Hill

Centers for Disease Control and Prevention

Prabasaj Paul

Centers for Disease Control and Prevention

Pragati V. Prasad

Centers for Disease Control and Prevention

Rachel B. Slayton

Centers for Disease Control and Prevention

\section{Research Article}

Keywords: 2019 novel coronavirus disease, COVID-19, SARS-CoV-2, coronavirus, mathematical modeling, severe acute respiratory syndrome coronavirus 2 , face masks.

Posted Date: October 5th, 2021

DOI: https://doi.org/10.21203/rs.3.rs-916637/v1

License: (c) (i) This work is licensed under a Creative Commons Attribution 4.0 International License.

Read Full License 
2 Title: Evaluation of Different Types of Face Masks to Limit the Spread of SARS-CoV-2 - A

3 Modeling Study

4 Authors: Brian M. Gurbaxani, $\mathrm{PhD}^{1 *}$; Andrew N. Hill, PhD ${ }^{2}$; Prabasaj Paul, PhD ${ }^{3}$; Pragati V.

$5 \quad$ Prasad, $\mathrm{MPH}^{3}$; Rachel B. Slayton, $\mathrm{PhD}^{3}$.

$6 \quad *$ These authors contributed equally to this work

7 Affiliations: Centers for Disease Control and Prevention, Atlanta, Georgia, USA

8 Article Summary Line: Adapting a published SARS-CoV-2 transmission model together with

9 updated, laboratory-derived source control and wearer protection efficacy estimates for a variety

10 of face coverings as well as N95 respirators, we demonstrate that community masking as

11 currently practiced has likely reduced cases and deaths and that this benefit can be increased with

12 wider adoption of better performing masks.

13 Running Title: Evaluation of Face Masks to Limit SARS-CoV-2

14 Keywords: 2019 novel coronavirus disease; COVID-19; SARS-CoV-2; coronavirus;

15 mathematical modeling; severe acute respiratory syndrome coronavirus 2; face masks.

16 All text: Times New Roman 12-pt. font; double-spaced and left aligned (left-justified).

17 Manuscript Number: 
22 We updated a published mathematical model of SARS-CoV-2 transmission with laboratory-

23 derived source and wearer protection efficacy estimates for a variety of face masks to estimate

24 their impact on COVID-19 incidence and related mortality in the United States. When used at

25 already-observed population rates of $80 \%$ for those $\geq 65$ years and $60 \%$ for those $<65$ years, face

26 masks are associated with $69 \%$ (cloth) to $78 \%$ (medical procedure mask) reductions in

27 cumulative COVID-19 infections and $82 \%$ (cloth) to $87 \%$ (medical procedure mask) reductions

28 in related deaths over a 6-month timeline in the model, assuming a basic reproductive number of

29 2.5. If cloth or medical procedure masks' source control and wearer protection efficacies are

30 boosted about $30 \%$ each to $84 \%$ and $60 \%$ by cloth over medical procedure masking, fitters, or

31 braces, the COVID-19 basic reproductive number of 2.5 could be reduced to an effective

32 reproductive number $\leq 1.0$, and from 6.0 to 2.3 for a variant of concern similar to delta

33 (B.1.617.2).

34

Text - (Word Count 3304)

36 Introduction:

37 The emergence of coronavirus disease 2019 (COVID-19) has had a substantial impact on populations globally, with efforts across governments to prevent its remarkable spread. While

39 social distancing has been universally recommended since very early in the pandemic,

40 recommendations for masks in the general population were adopted later in many countries (see,

41 for example, [1]). Several factors contributed to the initial uncertainty around the potential

42 impact of widespread use of face masks on SARS-CoV-2 transmission. A large and well-

43 designed 2015 study on cloth face masks (the main type of mask available to the public at the 
44 time) contributed to the scientific uncertainty that these types of face coverings were effective for

45 preventing the transmission of respiratory diseases [2]. There were initial hypotheses that cloth

46 masks could give the wearer a false sense of protection and even contaminate the wearer with

47 accumulated viral particles, notably described in a high-profile study in the Annals of Internal

48 Medicine that was later retracted (for failure to note PCR assay values that were below the limit

49 of detection) [3]. Furthermore, a major concern at the beginning of the outbreak in the US was

50 supply, especially of high-quality masks like N95 respirators. As it became clear, however, that

51 the virus can spread through exhaled respiratory droplets from infected individuals without

52 symptoms [4], the U.S. Centers for Disease Control and Prevention (CDC) recommended masks

53 for general use early in the U.S. pandemic (as of April 2020, [5]). Evidence continues to show

54 that asymptomatic and clinically mild infections contribute substantially to SARS-CoV-2

55 transmission [6-9]. Together, this growing body of evidence has highlighted the importance of

56 prevention measures, like masking, to reduce transmission from people who are asymptomatic,

57 undetected, or both.[6-8].

58 As the COVID-19 pandemic has continued, evidence has accumulated that face mask use by the

59 general population can limit the spread of SARS-CoV-2. This evidence has taken three main

60 forms, described in order of their appearance in the literature: 1) modeling studies that suggested

61 that even if masks are limited in their efficacy, widespread use across the population could still

62 reduce the spread of the virus to a considerable degree $[10,11], 2)$ laboratory studies that

63 demonstrated masks physically block exhaled droplets and aerosols containing virus from

64 infected persons (source control) and also offer wearer protection [12-14], and 3)

65 epidemiological studies that documented lower transmission in settings where masks were used

66 [15-19]. In this study, we extend the model of Worby and Chang to use age-stratified social 
67 contact patterns for the general U.S. population, and we analyzed the model both employing the measured face mask efficacy parameters for a variety of specific types of masks and for efficacy estimates that can act as benchmarks for evaluating these products [20].

\section{Methods:}

72 We adapted the transmission model (used for studying resource allocation of masks) of Worby 73 and Chang (2020) for face mask adoption in a hypothetical population by expanding it to the

74 age-stratified social contact patterns characteristic of the demographic profile of the United

75 States. The underlying structure of this compartmental model is described in Worby and Chang 76 [20], which we briefly summarize. Individuals are classified according to their disease status and

77 whether or not they wear a mask in public. The model is further stratified by age in 5-year age

78 bands. People contact each other (defined as either direct physical contact, e.g. through a

79 handshake or a kiss, or a proximal, two-way conversation of 3 or more words) at age-specific 80 daily rates estimated for the United States, as described by Mossong et al. and Prem et al. [21, 81 22]. We compared the results of the model with the age stratification removed, and the results

82 were significantly different (data not shown). Given that the infection fatality ratios (IFRs) are

83 strongly age structured, we believe the age stratification is appropriate. Vaccination is not 84 explicitly part of the model and has not been included in this study.

86 A schematic of the compartmental model is shown in Figure 1. Susceptible individuals who are

87 infected move into an exposed compartment and thereafter into a pre-symptomatic compartment.

88 Subsequently, a pre-specified proportion of these individuals moves into an asymptomatic state,

89 while the remainder become fully symptomatic. Pre-symptomatic, asymptomatic, and fully 
symptomatic SARS-CoV-2 infected individuals all contribute to the force of infection with

91 varying degrees of infectiousness. All asymptomatic individuals recover, whereas a proportion of

92 fully symptomatic individuals do not recover and die. A fraction of asymptomatic cases is

93 assumed to be detected whereupon a fraction of these individuals begins to use a mask and

94 continue to mask thereafter. Similarly, a fraction of symptomatic cases is assumed to know they

95 have COVID-19, and these individuals put on a mask at the same adoption level as detected in

96 asymptomatic cases. Symptomatic persons and detected, asymptomatic persons who wear a

97 mask also change their contact rates reflective of some degree of isolation/quarantine. We do not

98 include specific compartments modeling quarantine per se, but rather we reduce contact rates

99 which accomplishes the same purpose and maintains simplicity of compartmental structure while

100 allowing a degree of mixing that might be anticipated among a fraction of infected individuals

101 who are not strictly isolating themselves. We also assumed a fraction of the general population

102 adopts mask usage at the outset and continues usage regardless of infection status. Other than the

103 aforementioned masked cases, we assumed that contact rates among age groups remain the same

104 when people wear a mask. A basic reproduction number $\left(\mathrm{R}_{0}\right)$ of 2.5 was assumed in the absence

105 of any mask use, consistent with CDC's pandemic planning scenarios [23]. We also explored the

106 model with a basic reproduction number of 4.0, in keeping with the estimated magnitude of the

107 B.1.1.7 variant [24]. The modeled time horizon was 6 months and the cumulative number of

108 infections and deaths were recorded. The impact of various levels of mask adoption was assessed

109 by calculating the relative reduction in cumulative infection and deaths, comparing cumulative

110 cases and deaths to the same model over the same time horizon with no mask use in the entire

111 population. 
112 Masks were modeled to reduce transmission via two different mechanisms: source control

113 efficacy, whereby mask wearing by an infectious person reduces their likelihood of transmitting

114 SARS-CoV-2; and wearer protection efficacy, whereby masks protect a susceptible person from

115 becoming infected when exposed to an infectious person. We examined adoption of various

116 kinds of masks (e.g., cloth, medical procedure, N95 respirators) specifically incorporating

117 estimates from a recent study of source control efficacy [14]. A range of values of hypothetical

118 wearer protection efficacy was assumed for each kind of mask. Although it has generally been

119 found that wearer protectiveness coefficients are approximately half the source control values

$120[13,25,26]$, wearer protection efficacy was allowed to vary in the plot because it could be

121 greatly affected by how the mask is worn, maintained, and used. Characteristics of each mask

122 when worn according to manufacturers' specifications can be found in Lindsley et al. and are

123 shown in Table 1 [14]. We do not address the issue of mask and respirator use in healthcare

124 settings in this paper, as there is substantial public health guidance regarding the use of personal

125 protective equipment in healthcare settings [27].

126

127 Results:

128 Figure 2 depicts heat maps of reduced transmission and deaths over 6 months as a function of

129 varied source control efficacy and wearer protection efficacy. Mask wearing rates by the various

130 sub-populations in the model are provided in the figure caption. These rates were in line with

131 surveys of mask usage in the United States in May and June 2020 [28]. The colored bands of the

132 plots represent contours of relative reduction. Going from the bottom left corner of the figures

133 (source control efficacy and wearer protection efficacy both $0 \%$, equivalent to no mask wearing

134 in the population) these increase in 5\% increments to the right top corner (source control efficacy 
135 and wearer protection efficacy both 100\%). For example, to obtain at least a 50\% reduction in 136 cumulative infections, source control would need to be at least $55 \%$ efficacious in limiting 137 transmission in the population for arbitrary wearer protection efficacy. Source control would 138 need to be approximately $45 \%$ effective to reduce the number of deaths by half regardless of 139 wearer protection efficacy.

140 Even with the source control and wearer protection efficacy for the types of mask that most 141 wearers are likely to use, such as medical procedure or cloth masks and gaiters (see Table 1), 142 substantial reductions in case load and death can be achieved with general population use at 143 stated levels. Even at lower levels of use, reductions are estimated to be substantial As source 144 control and wearer protection efficacy approach $100 \%$ for the masks, relative reduction in 145 infections also approaches $100 \%$, even though mask adherence is far from $100 \%$, because 146 transmission dips below the epidemic threshold (i.e. an effective reproduction number $<1$ ). Our 147 simulations project that a $70 \%$ reduction in cumulative infections, relative to zero mask usage, 148 could be achieved with hypothetical combinations of wearer protection and source control 149 efficacies, respectively, of $(0 \%, 65 \%),(25 \%, 50 \%),(40 \%, 35 \%),(50 \%, 25 \%)$, among many 150 others lying on the $70 \%$ contour curve of the left panel of Figure 2.

151 Figure 3 depicts the reduction in infections with different population-wide percentages of mask 152 use, with the assumption that mask wearer protection efficacy is half of source control efficacy 153 and that mask use among persons $<65$ years old is $70 \%$ that of persons $\geq 65$ years old. We 154 evaluated these impacts for SARS-CoV-2 (3A, left) and one of its highly contagious variants of 155 concern (3B, right, for parameters similar to the Delta variant). Mask wearing rates for detected 156 and infected people are fixed at $90 \%$ for those $\geq 65$ years old, and $70 \%$ for those who are 157 younger. Based on the model, in Figure $3 \mathrm{~A}$ if $25 \%$ of the general population $\geq 65$ years old puts 
158 on a mask, cumulative cases after 6 months are reduced by $23 \%$ (N95), 14\% (medical

159 procedure), $12 \%$ (cloth), $12 \%$ (gaiter), and $9 \%$ (bandana). If mask adoption is $50 \%$ for the

160 general population $\geq 65$ years old, projected reductions in cases are $57 \%$ (N95), 32\% (medical

161 procedure), $28 \%$ (cloth), $28 \%$ (gaiter), and 20\% (bandana). If mask adoption is $75 \%$ for $\geq 65$

162 years old, projected reductions in cases are 95\% (N95), 65\% (medical procedure), 55\% (cloth),

$16354 \%$ (gaiter), and 35\% (bandana). Note that even with $0 \%$ mask use for the susceptible

164 population (horizontal axis), there is still a significant measure of infection control because of

165 mask adoption among detected infected people.

166 Figure 3B shows similar results to 3A, but assuming a much more highly contagious variant,

167 similar to Delta (B.1.617.2) with an $\mathrm{R} 0=6.0$. The results are dramatically different, and even a

168 high degree of adoption of the highest efficacy masks does not completely stop transmission.

169 Note that even if the susceptible population don masks at a $100 \%$ rate, the mask wearing rates of

170 detected asymptomatic and infected people are fixed at 90\% (for those $>65$ ) and $70 \%$ (for those

171 younger) in the simulation, which helps explain the seemingly low performance of $100 \%$ mask

172 wearing rate for N95 masks.

173 We estimated the incidence rate ratios (IRR) for new infections among mask wearers relative to

174 non-mask wearers over the course of 6 months, for different types of mask (Table 2). These

175 estimates reflect the impact of mask wearing on an individual wearer, whereas all of the other

176 analyses in this paper are focused on the population-level impact. The IRR at a given point in

177 time is the ratio of the number of new infections per capita among the mask wearing population

178 to the corresponding number among the non-mask wearing population. This assumes equal

179 mixing of masked and non-masked individuals - modeling the tendency for those populations to

180 self-segregate would tend to decrease these IRR values. As expected, the greater the mask 
181 efficacy, the greater the difference in new infection rates as measured by the IRR. After 6

182 months, new infections are projected to occur at around half the rate among mask wearers

183 compared to those not wearing N95 respirators, whereas in a scenario where medical procedure

184 masks are worn, infections among mask wearers occur at around a 32\% lower rate.

185 We evaluated the impact of face mask usage rates and efficacy parameters on the effective

186 reproduction number for $\mathrm{R}_{0}=2.5$ and $\mathrm{R}_{0}=6.0$, to represent the impact of highly contagious

187 variants of concern (e.g., B.1.617.2) (Figure 4) [24]. Note that warmer colors corresponding to

188 higher effective reproduction numbers are visible in the lower left-hand corner of the right panel

189 but less so in the left panel. As we approach 100\% source control and wearer protection

190 efficiencies, masks reduce effective reproduction number $<1$ for the low $\mathrm{R}_{0}$ scenario, but not for

191 the high $\mathrm{R}_{0}$ scenario, given the same wearing percentages used to generate Figure 2. For

192 example, when the baseline $\mathrm{R}_{0}=2.5$, an effective reproduction number of 1 is achieved by a

193 hypothetical mask with source control and wearer protection efficacies of $84 \%$ and $60 \%$,

194 respectively. However, these same efficacies would result in an effective reproduction number of

1952.33 when the baseline $\mathrm{R}_{0}=6.0$, as is likely the case with the Delta variant of concern. Those

196 efficacies for masks are achievable with common cloth masks and medical procedure masks if

197 they are doubled up, if the cloth masks have filter inserts, or if either type of mask is overfit with

198 a fitter or brace to ensure a tighter fit [29-31]. If source control efficacy is $96 \%$ and wearer

199 protection efficacy is $>70 \%$ (in line with efficacies for properly worn N95 respirators) then the

200 effective reproduction numbers $<1.0\left(\mathrm{R}_{0}=2.5\right)$ and $=2.19\left(\mathrm{R}_{0}=6.0\right)$. Similarly, adoption of

201 medical procedure masks (source control efficacy 56\%, wearer protection efficacy 28\%), results

202 in effective reproduction numbers of $1.30\left(\mathrm{R}_{0}=2.5\right)$ and $2.98\left(\mathrm{R}_{0}=6.0\right)$. Please note that in

203 Figure 4, even when source control and wearer protection efficacies of masks are zero, there is 
204 still some small measure of containment due to the reduced contact rates of those who are

205 detected and infected (whether symptomatic or asymptomatic) in the simulation.

\section{Discussion:}

208 Our results highlight the potential for substantial reduction in SARS-CoV-2 transmission, even

209 with moderately effective masks, when they are worn consistently correctly (over the chin and

210 covering nose and mouth) and/or per manufacturers' specifications by a large portion of the

211 population. These findings underscore the potential impact of population-wide measures that can

212 control transmission from infected individuals who do not have symptoms, both pre-

213 symptomatic individuals who are infectious prior to developing symptoms and individuals who

214 never experience symptoms. By extending the Worby and Chang model, we evaluated the

215 impact of different face mask use by age and highlight the need for wide adoption of these

216 interventions. Pairing this modeling framework with laboratory-derived parameters for source

217 control efficacy of different types of face masks helps to more accurately compare the relative

218 efficacy of each mask type as an intervention. Even with more specific source control

219 parameterization, the results are generally consistent with previous modeling studies [10, 11]:

220 face masks with realistic source control efficacy can reduce transmission substantially, and

221 widespread adoption can mitigate transmission at the population level. Furthermore, if the most

222 common types of face mask - cloth and medical procedure masks - can be enhanced with more

223 recent recommendations to improve fit around the nose and mouth, such as braces, elastic fitters,

224 or even double masking, those substantial reductions can be improved upon. 
226 Our study and several others suggest that the magnitude of reduction in SARS-CoV-2

227 transmission increases non-linearly with increased mask usage. The reasons for the non-linear

228 multiplier effect are several, at least including potential epidemiological, immunological, and

229 behavioral mechanisms $[17,27,31,32]$. Non-linear terms are inherent in the mathematical

230 mechanism of transmission reduction, given that masks act as both source control on the infected

231 and personal protection on the susceptible, terms which are multiplied together in the

232 transmission equations. This can be seen in the curvature of the line graphs of Figure 3 as mask

233 usage increases (diminishing returns can be seen as mask usage increases towards $100 \%$ in

234 Figure 3A for the N95 respirators, however). Furthermore, it is hypothesized that there are non-

235 linear effects inherent in the pathogenesis of SARS-CoV-2 infection, in that masks reduce the

236 initial viral exposure even if a wearer becomes infected despite the mask, decreasing the severity

237 of infection, reducing viral load and shedding, and increasing the asymptomatic ratio [17, 32 ,

238 33]. If this hypothesis is substantiated and we ignore complications arising from a higher

239 asymptomatic rate (i.e., more challenges with case identification), then there are potentially

240 several non-linear terms describing how the reproduction number decreases with mask efficacy

241 and use. Lastly, analysis of data on behavioral correlates of face mask use shows that people

242 wear face masks more often when they see others do so, even when they already intended to

243 wear a mask [28]. If changes in behavior were modeled, this would add another favorable non-

244 linear term to the impact of mask wearing.

246 The pandemic literature does contain a minority of reports that do not confirm the efficacy of

247 masks, although these studies have some important limitations. In particular, commentaries have

248 been written about the methodological limitations of a recent publication by Bundgaard et al. that 
249 appears to question the efficacy of face masks [34]. [35, 36]. Specifically, the study was only

250 powered to test if the wearer protection efficacy of medical procedure masks (referred to as

251 "surgical masks" in Bundgaard et al.) was $>50 \%$ and was not designed to measure their effect as

252 source control (because it was estimated only $5 \%$ of the population were wearing masks at the

253 time of the study). The Bundgaard et al. results were underpowered to detect wearer protection

254 efficacies of medical procedure and cloth masks. This is similar to another randomized

255 controlled trial (RCT) of cloth face masks as wearer protection against influenza virus infection

256 among healthcare workers by MacIntyre et al. [2]: the study was designed to evaluate only the

257 wearer protection effectiveness, not the source control effectiveness. Critically, the MacIntyre et

258 al. study did not compare cloth masks to no mask, only to masks of the health workers' choosing,

259 potentially including medical procedure masks. Hence, this RCT in a healthcare setting did not

260 have the negative control of not wearing a mask to help inform definitive conclusions about the

261 efficacy of cloth face masks for the general population in non-healthcare settings. In fact, a

262 follow-up study by MacIntyre et al. in 2020 found that healthcare workers whose cloth masks

263 were laundered by the hospital were protected as well as those who wore medical masks [37].

264 Also, recent results from an epidemiological study [38] analyzing population level mask

265 mandates where masks are more widely used are much more positive regarding the effectiveness

266 of masks.

267

268 Limitations:

269 Despite widespread usage of masks and other mitigation strategies [39], transmission of SARS-

270 CoV-2 remains inadequately controlled in the United States. There are many potential reasons

271 why surveillance data and ecologic field studies might not show the magnitude of reduction in 
272 infections due to increasing mask usage predicted here. The parameters used in the models

273 developed here might need to be better calibrated to match local transmission probabilities when

274 individuals contact one another (either through direct physical contact, e.g. through a handshake

275 or kiss, or a proximal, two-way conversation consisting of 3 or more words). Also, surveys

276 indicating mask usage in the population may have overestimated adherence over time or the

277 proper use or maintenance of masks. We model mask use as a set of parameters that can vary by

278 age, but not by other societal subgroups, and our age groups were only divided into $\geq 65$ years

279 and $<65$ years. Furthermore, our model does not distinguish between differing contact rates

280 within relevant populations such as schools, workplace, and households, but instead uses U.S.-

281 national estimates for contact rates.

282 The source data for mask efficacy used in these models were derived from controlled laboratory

283 simulations and not from human experiments. Measurements by other groups of filtration

284 efficiency using actual human volunteers tend to show more variation, and in some cases the

285 efficacies are lower than those reported here [40, 41].

286 Other limitations of the study are that mask usage is not assumed to vary over time, although it is

287 likely that consistent and correct mask use may increase or decrease over time as individuals

288 change their behaviors. Thus, we model homogeneous and unchanging mask use in a limited

289 number of subgroups vs. the reality that mask wearing is heterogeneous according to mask type,

290 sub-population, maintenance and proper use, and many other time-varying characteristics. This

291 may result in over-estimation of the impact of face masks on the pandemic. If so, even higher

292 mask uptake would be necessary to achieve substantial reductions in cases than is indicated here. 
293 Although the post-holiday 2020-2021 surge in cases seems large given a fairly high rate of mask

294 usage, we have no solid counterfactual information for comparison [12], i.e. we do not know

295 what the results would have been with no mask usage.

296 Conclusions:

297 Modeling studies, including this analysis, have estimated how face masks can reduce

298 transmission of SARS-CoV-2 and make a major impact at the population level, even with

299 varying levels of adherence and effectiveness of masks. Multiple public health interventions are

300 needed to reduce the transmission of SARS-CoV-2 and, as our analysis shows, robust use of face

301 masks is an important contributor. Face masks of various materials have the potential to

302 substantially reduce transmission in the SARS-CoV-2 pandemic, depending on the type and fit of

303 mask and the percentage adoption in the population. Furthermore, by attempting a more exact

304 quantitation of the impact of masking, studies like this can show, for example, that for highly

305 contagious new variants, such as the Delta variant of concern, masks alone are not enough to

306 contain the outbreak, and other control strategies are needed (e.g. social distancing, hand

307 washing, and vaccination). Public outreach and policies encouraging mask wearing, especially

308 highly efficacious masks, need to be encouraged along with other prevention strategies. In fact,

309 this study suggests that the current, imperfect use of masks has likely already reduced both cases

310 and deaths.

311

312

313 
314 Disclaimer: The findings and conclusions in this report are those of the authors and do not

315 necessarily represent the official position of the Centers for Disease Control and Prevention

316 (CDC).

317 Declarations:

318 Ethics approval and consent to participate: No human subjects were used in the study,

319 therefore no consent was needed.

320 Consent for publication: All authors consent to the publication. The paper has been cleared for

321 publication by the CDC. The pre-print version of this article is present on

322 https://www.medrxiv.org/content/10.1101/2021.04.21.21255889v1.

323 This article is not published nor is under publication elsewhere.

324 Availability of data and material: R code is available upon request.

325 Competing interests: All authors declare no competing interests.

326 Funding: All authors are employees of the CDC - no external funding was needed. 
327 Authors' contributions: BMG, ANH, and RBS conceived the study. BMG and ANH did the 328 background research and wrote the primary draft of the manuscript. ANH served as the primary

329 statistician for the project, adapted and modified the models and methods, and programmed the

330 models and equations in R. BMG and Prabasaj Paul served as secondary statisticians and

331 provided critical review of the models and equations, and BMG provided some ancillary models

332 and equations. BMG and ANH contributed equally to the paper. All authors helped pull

333 together the parameters needed for the models from the primary literature and other sources, and

334 all authors contributed to writing and critical review of the final manuscript.

335 Author details: ${ }^{1}$ Office of Science, Deputy Director for Public Health Science and Surveillance,

$336 \mathrm{CDC}$, and Departments of Electrical and Computer Engineering and Industrial and Systems

337 Engineering at the Georgia Institute of Technology; ${ }^{2}$ Division of Tuberculosis Elimination,

338 National Center for HIV/AIDS, Viral Hepatitis, STD, and TB Prevention, CDC, and Department

339 Biostatistics and Bioinformatics Department, Rollins School of Public Health, Emory University;

$340 \quad{ }^{3}$ CDC COVID-19 Response Team

341 Acknowledgements: The authors would like to thank all reviewers at the CDC who added to

342 the clarity of the final manuscript, as well as other members of the CDC COVID-19 Response

343 Modeling and Analytics Task Force for helpful discussions. 
348 Transmission of SARS-CoV-2 is modeled by a system of ordinary differential equations (ODE)

349 and compartments corresponding to age, disease status, and mask-wearing status. Compartments

350 comprise susceptible $(S)$, exposed $(E)$, pre-symptomatic $(P)$, asymptomatic and undetected $\left(A_{u}\right)$,

351 asymptomatic and detected $\left(A_{d}\right)$, symptomatic $(I)$, recovered $(R)$, and deceased $(D)$. The total

352 population is $N=S+E+P+A_{u}+A_{d}+I+R$. Initially, $N=100,000$. Each of these compartments

353 is further stratified by age (16 age groups from the POLYMOD study) and mask-wearing status

354 (yes/no). Thus, each disease compartment is represented by a $16 \times 2$ matrix, with entries

355 corresponding to the number of individuals of that particular disease status in age group $i=$

$3561, \ldots, 16$ and with mask status $j=0$ (no mask), 1 (mask). In matrix form, the ODE system is:

$$
\begin{aligned}
\dot{\boldsymbol{S}}= & -q\left\{M\left(\left(\boldsymbol{I}+p_{\text {rel inf }} \boldsymbol{A}_{d}\right) \cdot\left[\mathbf{1}_{16} r 1_{16}\right]+p_{\text {rel inf }}\left(\boldsymbol{P}+\boldsymbol{A}_{u}\right)\right) / N\left[\begin{array}{cc}
1 & 1-w \\
1-s & (1-w)(1-s)
\end{array}\right]\right\} \cdot \boldsymbol{S} \\
\dot{\boldsymbol{E}}= & q\left\{M\left(\left(\boldsymbol{I}+p_{\text {rel inf }} \boldsymbol{A}_{d}\right) \cdot\left[1_{16} r 1_{16}\right]+p_{\text {rel inf }}\left(\boldsymbol{P}+\boldsymbol{A}_{u}\right)\right) / N\left[\begin{array}{cc}
1 & 1-w \\
1-s & (1-w)(1-s)
\end{array}\right]\right\} \cdot \boldsymbol{S} \\
\dot{\boldsymbol{P}}= & \nu_{E} \boldsymbol{E}-\nu_{P} \boldsymbol{P} \\
\dot{\boldsymbol{A}}_{d}= & p_{\text {asym }} p_{\text {detect }} \nu_{P}\left(\boldsymbol{P}+\left(p_{\text {inf mask use }} \cdot \boldsymbol{P}\right)\left[\begin{array}{cc}
-1 & 1 \\
0 & 0
\end{array}\right]\right)-\nu_{A} \boldsymbol{A}_{d} \\
\dot{\boldsymbol{A}_{u}=} & p_{\text {asym }}\left(1-p_{\text {detect }}\right) \nu_{P} \boldsymbol{P}-\nu_{A} \boldsymbol{A}_{u} \\
\dot{\boldsymbol{I}}= & \left(1-p_{\text {asym }}\right) \nu_{P}\left(\boldsymbol{P}+\left(p_{\text {know }} p_{\text {inf mask use }} \cdot \boldsymbol{P}\right)\left[\begin{array}{cc}
-1 & 1 \\
0 & 0
\end{array}\right]\right)-\nu_{I} \boldsymbol{I} \\
\dot{\boldsymbol{R}}= & \nu_{A}\left(\boldsymbol{A}_{d}+\boldsymbol{A}_{u}\right)+\left(1-p_{\text {death }}\right) \nu_{I} \boldsymbol{I}
\end{aligned}
$$

Dot superscript denotes derivative with respect to time; central dot $\cdot$ indicates pointwise multiplication of matrices of the same dimension, or of the columns of a matrix by a vector of the same dimension. 
364 Compartment durations are specified by a rate $v_{J}$, where $J$ is the compartment. Average duration 365 in a compartment is $1 / v_{J}$. These rates model the durations of days exposed ( 2 days), pre-

366 symptomatic (4 days), asymptomatic ( 9 days), and symptomatic (9 days). Relative infectiousness

367 of pre-symptomatic and asymptomatic persons compared to symptomatic persons is $p_{\text {rel inf }}=0.75$.

368 Detection probability of an asymptomatic case is $p_{\text {detect }}=0.05$ and the proportion of

369 asymptomatic infections is $p_{\text {asym }}=0.30$. The risk of death for symptomatic individuals was

370 inferred from age-specific infection fatality ratios (IFR) via the equation $\mathrm{CFR}=\mathrm{IFR} /\left(1-p_{\text {asym }}\right)$,

371 where CFR denotes the case fatality ratio. IFRs are 0.00003 for ages $0-19$ years, 0.0002 ages 20-

$37249,0.005$ ages 50-69, 0.054 ages 70 and older. These parameters are based on the September

3732020 estimates included in the CDC Pandemic Planning Scenario \#5 [23]. We further assume

374 that $20 \%$ of symptomatic individuals know that that they are sick with SARS-CoV-2 and we

375 denote this fraction by $p_{\text {know. }}$ These people put on a mask.

376 POLYMOD daily contact rates were obtained from the study by Prem et al. [21]. The raw matrix

377 of contact rates was adjusted in the usual fashion to maintain balance (numbers of contacts of 378 age group $i$ with age group $j$ same as that of $j$ with $i$ ). The raw matrix $C$ is transformed to $C^{\prime}$ by

$$
C_{i j}^{\prime}=\frac{1}{2}\left(C_{i j}+C_{j i} N_{j} / N_{i}\right)
$$

382 where $N_{i}$ is the population size of age group $i$. Age distribution of the population was based on 383 American Community Survey (ACS) estimates for the U.S. population [42]. Matrix C' is, in 384 turn, transformed to give the symmetric matrix $M$ in the ODEs by 


$$
M_{i j}=\frac{N}{2}\left(C_{i j} / N_{j}+C_{j i} / N_{i}\right)=C_{i j}^{\prime} /\left(N_{j} / N\right)
$$

386 In this formulation, a typical term in the force of infection (FoI) arising from an age-stratified

387 infectious compartment $J=P, A_{d}, A_{u}$, $I$ of a given mask status is

$$
(M J)_{i} / N=\sum_{k=1}^{m} M_{i k} J_{k} / N=\sum_{k=1}^{m} C_{i k}^{\prime} J_{k} / N_{k}
$$

393 where $m=16$ is the number of POLYMOD age groups corresponding to 5-year age bands 394 ranging from younger than 5 years old to 70-75 years old, with the oldest age group comprising 395 persons 75 years and older. The FoI is further stratified in the ODEs above depending on mask status. This is implemented by the $2 \times 2$ matrix appearing in the differential equations for $S$ and

$397 E$. This matrix governs reductions in the FoI according to source control efficacy (sce, or $s$ for 398 brevity) and wearer protection efficiency (wpe, or w) conferred by the mask type. These combine 399 in four ways depending on the mask status of the infector and infectee (e.g., 1 - wpe and 1 - sce 400 multiply together in the case of transmission by mask wearers to mask wearers). We further 401 assume that symptomatic and detected, asymptomatic people who wear a mask have a lower, 402 daily rate of contact. As contact rates in compartmental models apply to susceptible, not 403 infectious, individuals, we model this as a reduction in infectiousness by a proportion $r$ for both 404 symptomatic and detected, asymptomatic mask-wearers. Specifically, if subscript $i$ indexes age 405 group and superscripts 'none' and 'mask' denote no mask and mask wearing, respectively, 406 expanding the matrix formulation of the ODEs for susceptible individuals gives 


$$
\begin{aligned}
\dot{S}_{i}^{\text {none }} & =-q \sum_{k=1}^{m} M_{i k}\left(K_{k}^{\text {none }}+(1-\text { sce }) K_{k}^{\text {mask }}\right) \times S_{i}^{\text {none }} / N \\
\dot{S}_{i}^{\text {mask }} & =-q(1-\text { wpe }) \sum_{k=1}^{m} M_{i k}\left(K_{k}^{\text {none }}+(1-\text { sce }) K_{k}^{\text {mask }}\right) \times S_{i}^{\text {mask }} / N
\end{aligned}
$$

where $K^{\text {none }}=I^{\text {none }}+p_{\text {rel inf }}\left(P^{\text {none }}+A_{d}^{\text {none }}+A_{u}^{\text {none }}\right)$ and

$K^{\text {mask }}=r I^{\text {mask }}+p_{\text {rel inf }}\left(P^{\text {mask }}+r A_{d}^{\text {mask }}+A_{u}^{\text {mask }}\right)$.

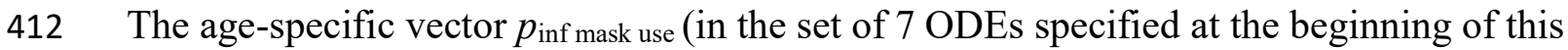

413 supplement) specifies the proportion of symptomatic and detected asymptomatic people who don

414 a mask upon learning they are infectious. The corresponding proportion for symptomatic

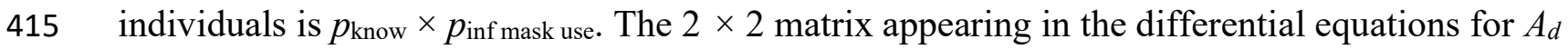

416 and $I$ governs the adoption of masks by asymptomatic individuals when they are detected (the

417 first column corresponds to no mask, the second to mask wearing). In simulations, we assumed

418 that individuals aged 65 years and older adopted masks at one proportion and younger than 65

419 years at another, lower, proportion (but this can be changed by the user). Expanding the matrix

420 formulation of the ODEs for detected asymptomatic individuals gives

$$
\begin{aligned}
& \dot{A}_{d, i}^{\text {none }}=p_{\text {asym }} p_{\text {detect }} \nu_{P}\left(1-p_{\text {inf mask use }, i}\right) P_{i}^{\text {none }}-\nu_{A} A_{d, i}^{\text {none }} \\
& \dot{A}_{d, i}^{\text {mask }}=p_{\text {asym }} p_{\text {detect }} \nu_{P}\left(P_{i}^{\text {mask }}+p_{\text {inf mask use }, i} P_{i}^{\text {none }}\right)-\nu_{A} A_{d, i}^{\text {mask }}
\end{aligned}
$$

422 It is assumed that a proportion of the general population (susceptible individuals) wear a mask at 423 the outset and keep it on at all times (or at least when mixing in the population). This proportion 424 can vary by age. We assume that in the general population, $80 \%$ of those aged 65 and older and $42560 \%$ of the rest, wear a mask (and keep it on indefinitely). 
426 To seed the epidemic, we arbitrarily assumed that there were 10 detected, asymptomatic non-

427 masked individuals in each age group at the outset. Time units were expressed in days. The

428 model was run for 6 months (183 days) with a timestep of 0.25 days using a Runge-Kutta solver

429 in R v.3.6.3 [43] using the package 'deSolve' [44].

430 The FoI was calibrated to yield a basic reproduction number $R_{0}=2.5$ for the sub-model without

431 mask usage. This yielded the parameter $q=0.01429$, which represents the probability of a

432 symptomatic infectious person infecting a susceptible person upon contact between them. The

433 reproduction number was calculated as the dominant eigenvalue of the next-generation matrix

434 (NGM) using the method of van den Driessche and Watmough [45]. Computation was facilitated

435 by the R package 'blockmatrix' [46] owing to the sparseness of the matrices involved. Details of 436 this calculation are described further below.

437 Calculation of $R_{0}$

438 Following [45], we construct matrices $F$, describing rates at which infectious individuals produce 439 new infections, and $V$, consisting of all other rates, whose inverse describes average durations in 440 compartments. The $i$ th row and $j$ th column of these matrices is the partial derivative of the right441 hand side of the differential equation for compartment $i$ with respect to compartment $j$, evaluated 442 at the disease-free equilibrium (DFE). Only the 5 infected compartment types are considered, 443 namely, $E, P, A_{d}, A_{u}, I$, enumerated by age group and mask status. The basic reproduction 444 number $R_{0}$ is given by the dominant eigenvalue of the NGM $F V^{-1}$.

445 Matrices $F$ and $V$ are of dimension $160 \times 160(2$ mask statuses $\times 16$ age groups $\times 5$ relevant 446 compartment types). However, as new infections only arise from the $E$ compartments, via the 447 previously described FoI, matrix $F$ is sparse. So too is $V$, as a lot of its sub-blocks are zero or 
448 diagonal matrices. Hence, we can construct these matrices in block form. We use the Kronecker 449 product of matrices, which we denote by $\otimes$.

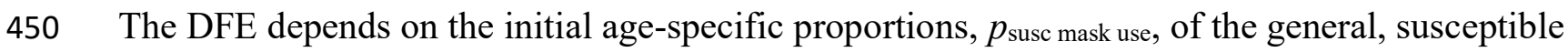
451 population wearing a mask. Multiplying the ACS age group proportions pointwise by $p_{\text {susc mask use }}$ 452 and $1-p_{\text {susc mask use }}$ gives the age-specific proportions of the population with and without masks, 453 respectively. These are multiplied by the hypothetical total population size $N=100,000$ to obtain 454 numbers in each stratum.

455 Vectorizing the $16 \times 2$ matrices in the ODEs by stacking columns into a single $32 \times 1$ column 456 vector, we have the following constituent matrices for calculating $R_{0}$ :

$$
\begin{aligned}
& F_{1}=\underbrace{\left[\begin{array}{l}
1 \\
0 \\
0 \\
0 \\
0
\end{array}\right]}_{5 \times 1} \otimes\{(\underbrace{\left[\begin{array}{c}
1 \\
1-w
\end{array}\right]}_{2 \times 1} \otimes \underbrace{\left[0 p_{\text {ri }} p_{\text {ri }} p_{\text {ri }} 1\right]}_{1 \times 5}] \otimes \underbrace{[11-s]}_{1 \times 2} \cdot \underbrace{G}_{2 \times 10}\} \otimes \underbrace{\left(M \cdot p_{\text {ACS }}\right)}_{16 \times 16}) \cdot \underbrace{\left.\begin{array}{c}
1_{16}-p_{\text {smu }} \\
p_{\text {smu }}
\end{array}\right]}_{32 \times 1}\} \\
& G=\left[\begin{array}{llll}
\mathbf{1}_{5}^{\top} & r & 1_{3}^{\top} & r \\
1_{5}^{\top} & r & 1_{3}^{\top} & r
\end{array}\right]
\end{aligned}
$$

458 For brevity, $p_{\text {r.i. }}$ denotes $p_{\text {rel inf }}$ above, $p_{\text {ACS }}$ is the ACS population age distribution, $p_{\text {s.m.u. }}$ denotes

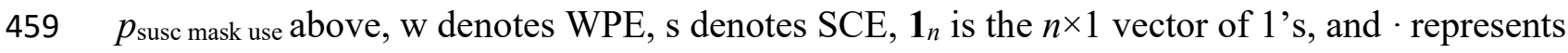

460 pointwise multiplication by column. Matrix $M$ is the POLYMOD-derived contact matrix

461 described earlier. It follows from the definition of Kronecker product that $F_{1}$ is $160 \times 160$. The

462 order of entries in row or column vectors of length 5 corresponds to the compartment types $E$, $P$,

$463 A_{d}, A_{u}, I$. Thus, the $5 \times 1$ vector on the left represents new infections only arising from

464 compartments of type $E$ (component 1) and not from $P, A_{d}, A_{u}, I$ (components 2 to 5 ) and its

465 occurrence renders $F_{1}$ sparse. The $1 \times 5$ row vector has components $2-4$ as $p_{\text {rel inf, indicating the }}$

466 relative infectiousness of presymptomatic and detected and undetected asymptomatic individuals 
$467\left(P, A_{d}, A_{u}\right)$ compared to symptomatic individuals $I$ (component 5). Row and column vectors of

468 length 2 correspond to mask efficacies. The vector $p_{\text {ACS }}$ represents the population age-

469 distribution and the $32 \times 1$ vector on the right denotes the age-specific general population mask

470 wearing proportions.

471 The matrix $F$ is given by $F=q F_{1}$, where $q$ is the calibration parameter representing the

472 probability of symptomatic infectious persons infecting susceptible persons upon contact

473 between them.

474 Matrix $V$ may also be expressed in block form as:

475

476

$$
V=\left[\begin{array}{ccccc}
V_{E E} & 0 & 0 & 0 & 0 \\
V_{P E} & V_{P P} & 0 & 0 & 0 \\
0 & V_{A_{d} P} & V_{A_{d} A_{d}} & 0 & 0 \\
0 & V_{A_{u} P} & 0 & V_{A_{u} A_{u}} & 0 \\
0 & V_{I P} & 0 & 0 & V_{I I}
\end{array}\right]
$$

477 where each block is a $32 \times 32$ matrix. Theory guarantees $V$ is invertible. As with $F$, the order of

478 the component types in block rows and columns here is $E, P, A_{d}, A_{u}, I$. The nonzero blocks are as

479 follows, with parameter notation as given earlier, and $I_{n}$ denoting the $n \times n$ identity matrix:

$$
\begin{aligned}
V_{E E} & =\nu_{E} I_{32} \\
V_{P E} & =-\nu_{E} I_{32} \\
V_{P P} & =\nu_{P} I_{32} \\
V_{A_{d} P} & =-\nu_{P} p_{\text {detect }} p_{\text {asym }} B_{A} \\
\text { where } B_{A} & =\left[\begin{array}{cc}
\operatorname{diag}\left(1-p_{\text {inf mask use }}\right) & 0 \\
\operatorname{diag}\left(p_{\text {inf mask use }}\right) & I_{16}
\end{array}\right](16 \times 16 \text { blocks }) \\
V_{A_{d} A_{d}} & =\nu_{A} I_{32} \\
V_{A_{u} P} & =-\nu_{P}\left(1-p_{\text {detect }}\right) p_{\text {asym }} I_{32} \\
V_{A_{u} A_{u}} & =\nu_{A} I_{32} \\
V_{I P} & =-\nu_{P}\left(1-p_{\text {asym }}\right) B_{I} \\
\text { where } B_{I} & =\left[\begin{array}{cc}
\operatorname{diag}\left(1-p_{\text {know }} p_{\text {inf mask use }}\right) & 0 \\
p_{\text {know }} \operatorname{diag}\left(p_{\text {inf mask use }}\right) & I_{16}
\end{array}\right](16 \times 16 \text { blocks }) \\
V_{I I} & =\nu_{I} I_{32}
\end{aligned}
$$


481 Blocks $V_{X X}$ on the leading diagonal correspond to outflows from compartment type $X$. Off-

482 diagonal blocks $V_{X Y}$ correspond to inflows from compartment type $Y$ to compartment type $X$. The

$483 B$ matrices correspond to the adoption of mask use (change of mask status) upon asymptomatic

484 detection/symptomatic awareness, according to the age-specific proportion ( $p_{\text {inf mask use }}$ ) who do

485 so. Denoting the dominant eigenvalue of a matrix by $\rho$, we have

$486 \quad R_{0}=\rho\left(F V^{-1}\right)=q \times \rho\left(F_{1} V^{-1}\right)$.

487 Setting baseline $R_{0}=2.5$ (no mask use), we calibrate $q=2.5 \div \rho\left(F_{1} V^{-1}\right.$ ).

488 Computation of symptomatic and asymptomatic detection rates

489 The percentage of symptomatic and asymptomatic SARS-CoV-2 cases that are detected are not

490 established numbers, but they are suspected to be low given the general detection rate of $16.1 \%$

$491[47,48]$. Both can be estimated with a simple Bayesian calculation, however, given the general

492 detection rate (P(case) in the equation below), the asymptomatic rate of infections of 0.3 [23],

493 and the probability that detected cases are and remain asymptomatic $(0.2)$ or symptomatic $(0.8)$

494 [49]. For example, the probability that a person becomes a detected case (e.g. through contact

495 tracing efforts) given the person is asymptomatic is given below

$496 \mathrm{P}($ case $\mid$ asymptomatic $)=\mathrm{P}($ asymptomatic $\mid$ case $) \mathrm{P}($ case $) / \mathrm{P}($ asymptomatic $)$

497 The simple calculation yields a $10.7 \%$ detection rate for asymptomatic individuals, and a $18.3 \%$

498 detection rate for those with symptoms. The $18.3 \%$ figure appears to be in line with

499 epidemiological estimates as well [50].

500

References:

501 
1. Brosseau, L.M., and Sietsema, M., Commentary: Masks-For-All for Covid-19 Not Based on Sound Data (University of Minnesota). 2020.

2. Maclntyre, C.R., et al., A cluster randomised trial of cloth masks compared with medical masks in healthcare workers. BMJ Open, 2015. 5(4): p. e006577.

3. Bae, S., et al., Effectiveness of Surgical and Cotton Masks in Blocking SARS-CoV-2: A Controlled Comparison in 4 Patients. Ann Intern Med, 2020. 173(1): p. W22-w23.

4. Bai, Y., et al., Presumed Asymptomatic Carrier Transmission of COVID-19. JAMA, 2020. 323(14): p. 1406-1407.

5. Centers for Disease Control and Prevention. Considerations for Wearing Masks. 2020 December 18, 2020; Available from: https://www.cdc.gov/coronavirus/2019-ncov/prevent-gettingsick/cloth-face-cover.html.

6. Byambasuren, O., et al., Estimating the extent of asymptomatic COVID-19 and its potential for community transmission: systematic review and meta-analysis. JAMMI, 2020.

7. Poletti, P., et al., Probability of symptoms and critical disease after SARS-CoV-2 infection. arXiv.

8. Oran, D.P. and E.J. Topol, Prevalence of Asymptomatic SARS-CoV-2 Infection : A Narrative Review. Ann Intern Med, 2020. 173(5): p. 362-367.

9. Johansson, M.A., et al., SARS-CoV-2 Transmission From People Without COVID-19 Symptoms. JAMA Network Open, 2021. 4(1): p. e2035057-e2035057.

10. Ngonghala, C.N., et al., Mathematical assessment of the impact of non-pharmaceutical interventions on curtailing the 2019 novel Coronavirus. Math Biosci, 2020. 325: p. 108364.

11. Eikenberry, S.E., et al., To mask or not to mask: Modeling the potential for face mask use by the general public to curtail the COVID-19 pandemic. Infect Dis Model, 2020. 5: p. 293-308.

12. Fisher, K.A., et al., Factors Associated with Cloth Face Covering Use Among Adults During the COVID-19 Pandemic - United States, April and May 2020. MMWR Morb Mortal Wkly Rep, 2020. 69(28): p. 933-937.

13. Konda, A., et al., Aerosol Filtration Efficiency of Common Fabrics Used in Respiratory Cloth Masks. ACS Nano, 2020. 14(5): p. 6339-6347.

14. Lindsley, W.G., et al., Efficacy of face masks, neck gaiters and face shields for reducing the expulsion of simulated cough-generated aerosols. Aerosol Science and Technology, 2020: p. 112.

15. Payne, D.C., et al., SARS-CoV-2 Infections and Serologic Responses from a Sample of U.S. Navy Service Members - USS Theodore Roosevelt, April 2020. MMWR Morb Mortal Wkly Rep, 2020. 69(23): p. 714-721.

16. Hendrix, M.J., et al., Absence of Apparent Transmission of SARS-CoV-2 from Two Stylists After Exposure at a Hair Salon with a Universal Face Covering Policy - Springfield, Missouri, May 2020. MMWR Morb Mortal Wkly Rep, 2020. 69(28): p. 930-932.

17. Ing, A.J., C. Cocks, and J.P. Green, COVID-19: in the footsteps of Ernest Shackleton. Thorax, 2020. 75(8): p. 693-694.

18. Lyu, W. and G.L. Wehby, Community Use Of Face Masks And COVID-19: Evidence From A Natural Experiment Of State Mandates In The US. Health Aff (Millwood), 2020. 39(8): p. 1419-1425.

19. Brooks, J.T. and J.C. Butler, Effectiveness of Mask Wearing to Control Community Spread of SARS-CoV-2. Jama, 2021. 325(10): p. 998-999.

20. Worby, C.J. and H.H. Chang, Face mask use in the general population and optimal resource allocation during the COVID-19 pandemic. Nat Commun, 2020. 11(1): p. 4049.

21. Prem, K., A.R. Cook, and M. Jit, Projecting social contact matrices in 152 countries using contact surveys and demographic data. PLoS Comput Biol, 2017. 13(9): p. e1005697.

22. Mossong, J., et al., Social contacts and mixing patterns relevant to the spread of infectious diseases. PLoS medicine, 2008. 5(3): p. e74-e74. 
23. Centers for Disease Control and Prevention. COVID-19 Pandemic Planning Scenarios. 2020 September 10, 2020; Available from: https://www.cdc.gov/coronavirus/2019ncov/hcp/planning-scenarios.html.

24. Volz, E., et al., Transmission of SARS-CoV-2 Lineage B.1.1.7 in England: Insights from linking epidemiological and genetic data. 2021: p. 2020.12.30.20249034.

25. Chan, J.F., et al., Surgical Mask Partition Reduces the Risk of Noncontact Transmission in a Golden Syrian Hamster Model for Coronavirus Disease 2019 (COVID-19). Clin Infect Dis, 2020. 71(16): p. 2139-2149.

26. Ueki, H., et al., Effectiveness of Face Masks in Preventing Airborne Transmission of SARS-CoV-2. mSphere, 2020. 5(5).

27. Centers for Disease Control and Prevention. Interim Infection Prevention and Control Recommendations for Healthcare Personnel During the Coronavirus Disease 2019 (COVID-19) Pandemic. 2020 December 14, 2020; Available from: Interim Infection Prevention and Control Recommendations for Healthcare Personnel During the Coronavirus Disease 2019 (COVID-19) Pandemic.

28. Barile, J.P., et al., Theory-based Behavioral Predictors of Self-reported Use of Face Coverings in Public Settings during the COVID-19 Pandemic in the United States. Ann Behav Med, 2020.

29. Gandhi, M. and L.C. Marr, Uniting Infectious Disease and Physical Science Principles on the Importance of Face Masks for COVID-19. Med, 2021. 2(1): p. 29-32.

30. Rothamer, D.A., et al., Strategies to minimize SARS-CoV-2 transmission in classroom settings: Combined impacts of ventilation and mask effective filtration efficiency. 2021: $p$. 2020.12.31.20249101.

31. Brooks, J.T., et al., Maximizing Fit for Cloth and Medical Procedure Masks to Improve Performance and Reduce SARS-CoV-2 Transmission and Exposure, 2021. MMWR Morb Mortal Wkly Rep, 2021. 70(7): p. 254-257.

32. Gandhi, M. and G.W. Rutherford, Facial Masking for Covid-19-Potential for "Variolation" as We Await a Vaccine. N Engl J Med, 2020. 383(18): p. e101.

33. Sims, M.D., et al., COVID-19 seropositivity and asymptomatic rates in healthcare workers are associated with job function and masking. Clin Infect Dis, 2020.

34. Bundgaard, H., et al., Effectiveness of Adding a Mask Recommendation to Other Public Health Measures to Prevent SARS-CoV-2 Infection in Danish Mask Wearers : A Randomized Controlled Trial. Ann Intern Med, 2020.

35. Laine, C., S.N. Goodman, and E. Guallar, The Role of Masks in Mitigating the SARS-CoV-2 Pandemic: Another Piece of the Puzzle. Ann Intern Med, 2020.

36. Frieden, T.R. and S. Cash-Goldwasser, Of Masks and Methods. Ann Intern Med, 2020.

37. Maclntyre, C.R., et al., Contamination and washing of cloth masks and risk of infection among hospital health workers in Vietnam: a post hoc analysis of a randomised controlled trial. BMJ Open, 2020. 10(9): p. e042045.

38. Van Dyke, M.E., et al., Trends in County-Level COVID-19 Incidence in Counties With and Without a Mask Mandate - Kansas, June 1-August 23, 2020. MMWR Morb Mortal Wkly Rep, 2020. 69(47): p. 1777-1781.

39. Hutchins, H.J., et al., COVID-19 Mitigation Behaviors by Age Group - United States, April-June 2020. MMWR Morb Mortal Wkly Rep, 2020. 69(43): p. 1584-1590.

40. Clapp, P.W., et al., Evaluation of Cloth Masks and Modified Procedure Masks as Personal Protective Equipment for the Public During the COVID-19 Pandemic. JAMA Intern Med, 2021. 181(4): p. 463-469. 
41. Mueller, A.V., et al., Quantitative Method for Comparative Assessment of Particle Removal Efficiency of Fabric Masks as Alternatives to Standard Surgical Masks for PPE. Matter, 2020. 3(3): p. 950-962.

42. U.S. Census Bureau, American Community Survey, T. S0101, Editor. 2019, U.S. Census Bureau: data.census.gov.

43. R Core Development Team, A language and environment for statistical computing., R.F.f.S. Computing., Editor. 2020.

44. Soetaert, K., T. Petzoldt, and R.W. Setzer, Solving Differential Equations in R: Package deSolve. 2010, 2010. 33(9): p. 25 \%J Journal of Statistical Software.

45. van den Driessche, P. and J. Watmough, Reproduction numbers and sub-threshold endemic equilibria for compartmental models of disease transmission. Math Biosci, 2002. 180: p. 29-48.

46. Cordano, E., blockmatrix: Tools to solve algebraic systems with partitioned matrices. R package version 1.0. 2014.

47. Alroy, K.A., et al., Population-Based Estimates of Coronavirus Disease 2019 (COVID-19)-like IIIness, COVID-19 IIIness, and Rates of Case Ascertainment, Hospitalizations, and DeathsNoninstitutionalized New York City Residents, March-April 2020. Clinical Infectious Diseases, 2021.

48. Phipps, S.J., R.Q. Grafton, and T. Kompas, Robust estimates of the true (population) infection rate for COVID-19: a backcasting approach. R Soc Open Sci, 2020. 7(11): p. 200909.

49. Buitrago-Garcia, D., et al., Occurrence and transmission potential of asymptomatic and presymptomatic SARS-CoV-2 infections: A living systematic review and meta-analysis. PLOS Medicine, 2020. 17(9): p. e1003346.

50. Smith, L.E., et al., Adherence to the test, trace, and isolate system in the UK: results from 37 nationally representative surveys. BMJ, 2021. 372: p. n608.

51. Li, B., et al., Viral infection and transmission in a large, well-traced outbreak caused by the SARSCoV-2 Delta variant. medRxiv, 2021: p. 2021.07.07.21260122.

52. Zhang, M., et al., Transmission Dynamics of an Outbreak of the COVID-19 Delta Variant B.1.617.2 - Guangdong Province, China, May - June 2021. China CDC Weekly, 2021. 3: p. 584-586.

53. Herlihy, R., et al., Rapid Increase in Circulation of the SARS-CoV-2 B.1.617.2 (Delta) Variant Mesa County, Colorado, April-June 2021. MMWR Morb Mortal Wkly Rep, 2021. 70(32): p. 10841087.

54. European Centre for Disease Prevention and Control, Implications for the EU/EEA on the spread of the SARSCoV-2 Delta (B.1.617.2) variant of concern - 23 June 2021. 2021, ECDC: Stockholm, Sweden.

Address for Correspondence:

Brian M. Gurbaxani, PhD. Address: 1600 Clifton Rd NE, Atlanta, GA 30333. Email:

buw8@cdc.gov.

Andrew N. Hill, PhD. Address: 1600 Clifton Rd NE, Atlanta, GA 30333. Email: fyu7@,cdc.gov.

Tables

Table 1: Parameter values used in the simulation 


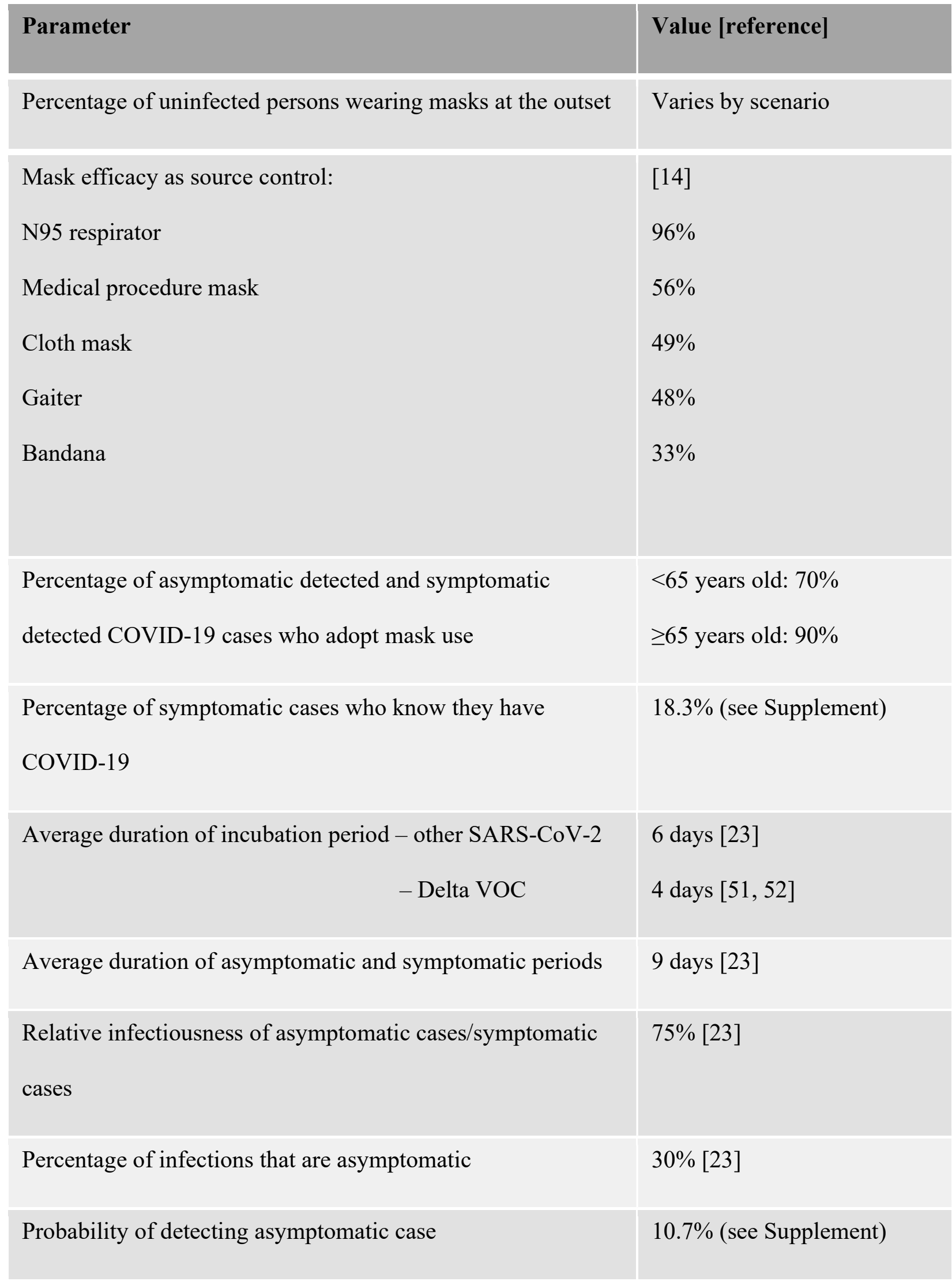


Infection Fatality Ratio (IFR)

(IFR's for Delta VOC $\approx 2 \mathrm{x}$ shown for other SARS-CoV-2)

$[53,54]$

Reduction in contact rate for symptomatic and detected

asymptomatic persons wearing a mask

Risk of death for symptomatic cases
Ages 0-19: 0.00003

Ages 20-49: 0.0002

Ages 50-69: 0.005

Ages 70+: 0.054 [23]

$50 \%$

See Supplement for

calculation

637 Table 2: Incidence rate ratios (IRR) at 2-month intervals of new infections among masked vs.

638 non-masked population. Each row represents a scenario in which all mask-wearing individuals

639 are assumed to wear the specified type of mask. Wearer protection efficacy is assumed to be half

640 of source control efficacy. It assumes $60 \%$ of the susceptible population $<65$ years old are

641 wearing masks, $80 \%$ of those $\geq 65$ years old wear masks, and both rates increase $10 \%$ for both

642 detected and infected persons (whether symptomatic or asymptomatic).

\begin{tabular}{|l|r|r|r|}
\hline \multicolumn{3}{|c|}{2 months } & \multicolumn{2}{|c|}{4 months 6 months } \\
\hline Type of & \multicolumn{3}{|c|}{ IRR } \\
Mask & & & \\
\hline N95 & & 0.47 & 0.47 \\
\hline respirator & 0.47 & & \\
\hline Medical & & 0.66 & 0.68 \\
\hline procedure & 0.66 & & \\
\hline
\end{tabular}




\begin{tabular}{|l|r|r|r|}
\hline Cloth & & & \\
\hline Mask & 0.69 & 0.7 & 0.72 \\
\hline Gaiter & 0.69 & 0.7 & 0.72 \\
\hline Bandana & 0.76 & 0.78 & 0.8 \\
\hline
\end{tabular}

Figure Legends

645 Figure 1: Schematic of compartmental model. Compartments are susceptible (S, green), 646 exposed (E, yellow), infectious compartments (pre-symptomatic P, asymptomatic and detected $647 \mathrm{~A}_{\mathrm{d}}$, asymptomatic and undetected $\mathrm{A}_{\mathrm{u}}$, symptomatic I, pink), recovered ( $\mathrm{R}$, gray), and died (D, 648 gray). Superscript 'n' denotes no mask, and 'm' denotes mask.

649 Figure 2: Heat maps of the percentage reduction in cumulative infections at the end of 1 year 650 relative to no mask use in the population, assuming a baseline $\mathrm{R} 0=2.5$. Assumes $60 \%$ of the 651 susceptible population $<65$ years old are wearing masks, $80 \%$ of those $\geq 65$ years old wear 652 masks, and both rates increase $10 \%$ for detectably infected persons (whether symptomatic or 653 asymptomatic). The simulation posits that $18.3 \%$ of symptomatic infected people and $10.7 \%$ of 654 asymptomatic infected individuals have been detected by screening and are known to be carrying 655 SARS-CoV-2 (see the Supplement). Mask efficacy parameters for source control and wearer 656 protection increase along the vertical and horizontal axes, respectively. Reductions in 657 cumulative infections over 6 months are shown on the left; reductions in deaths are shown on the 658 right.

659 Figure 3: The percentage reduction in cumulative infections after 6 months of simulation, 660 relative to no mask use in the population, as mask use varies in the general, susceptible 661 population for different types of face masks. Mask source control parameters are fixed 
662 according to estimates for the given types, and wearer protection efficiency is assumed to be half

663 of source control effectiveness. In this analysis, younger susceptible persons are assumed to use

664 masks at $70 \%$ of the rate of persons $\geq 65$ years old. Known infected people $\geq 65$ years old are

665 masked at a $90 \%$ rate, with younger persons at $70 \%$. The baseline $\mathrm{R}_{0}$ in the absence of mask use

666 is assumed to be 2.5 in the left panel and 6.0 in the right panel.

667 Figure 4: Effective reproduction number for given mask use by varying efficacy parameters

668 shown on the horizontal and vertical axes. This analysis assumes $90 \%$ and $70 \%$ mask use rates

669 for infectious and detected persons older and younger than 65 years of age, respectively, and

$67080 \%$ and $60 \%$ among susceptible persons for the same age breakdown. Asymptomatic detection

671 and symptomatic awareness fractions are given in Table 1 . The baseline $\mathrm{R}_{0}$ in the absence of

672 mask use is assumed to be 2.5 in the left panel and 6.0 in the right panel.

673 


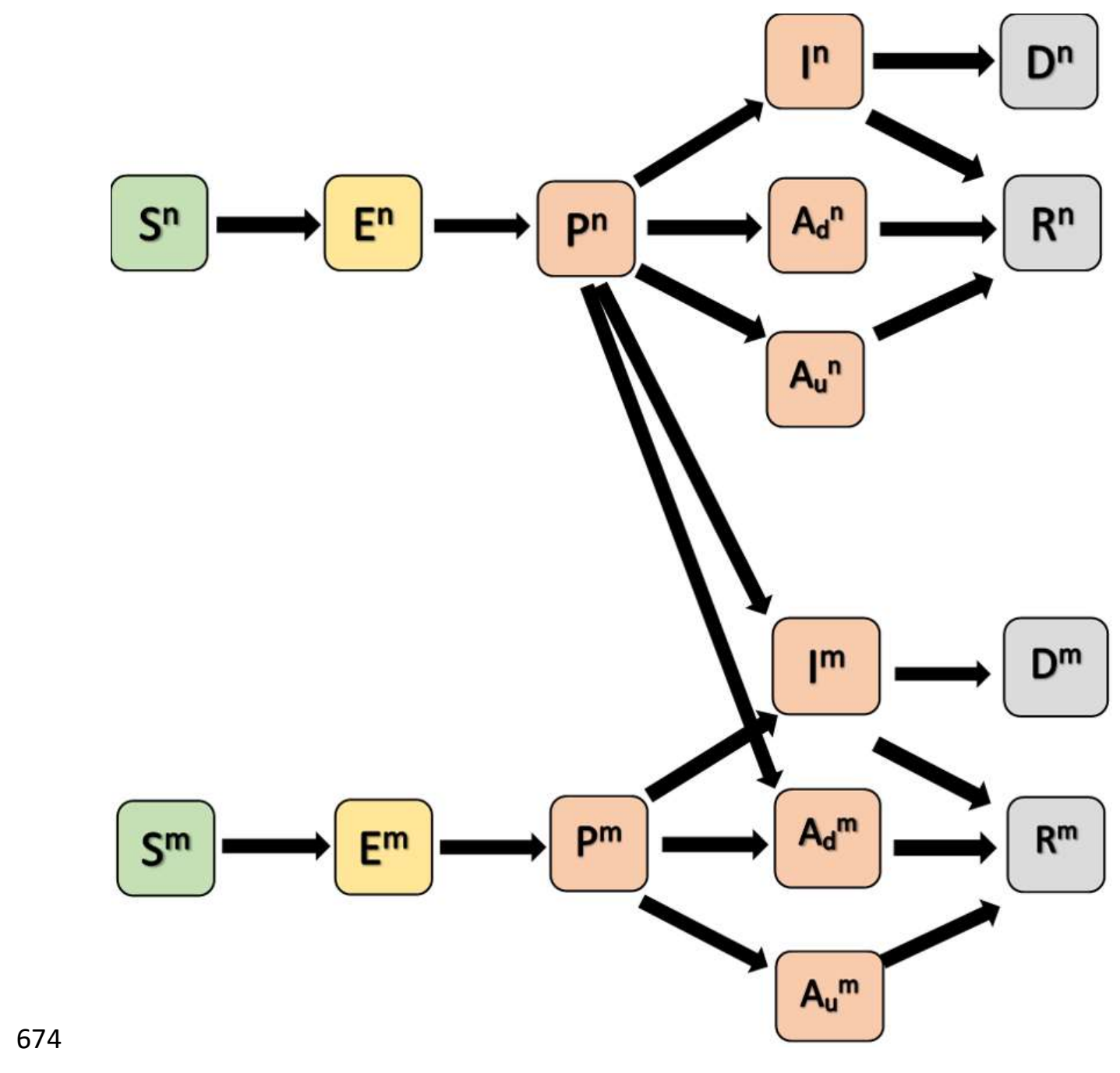

675

$676 \quad$ Figure 1

677 

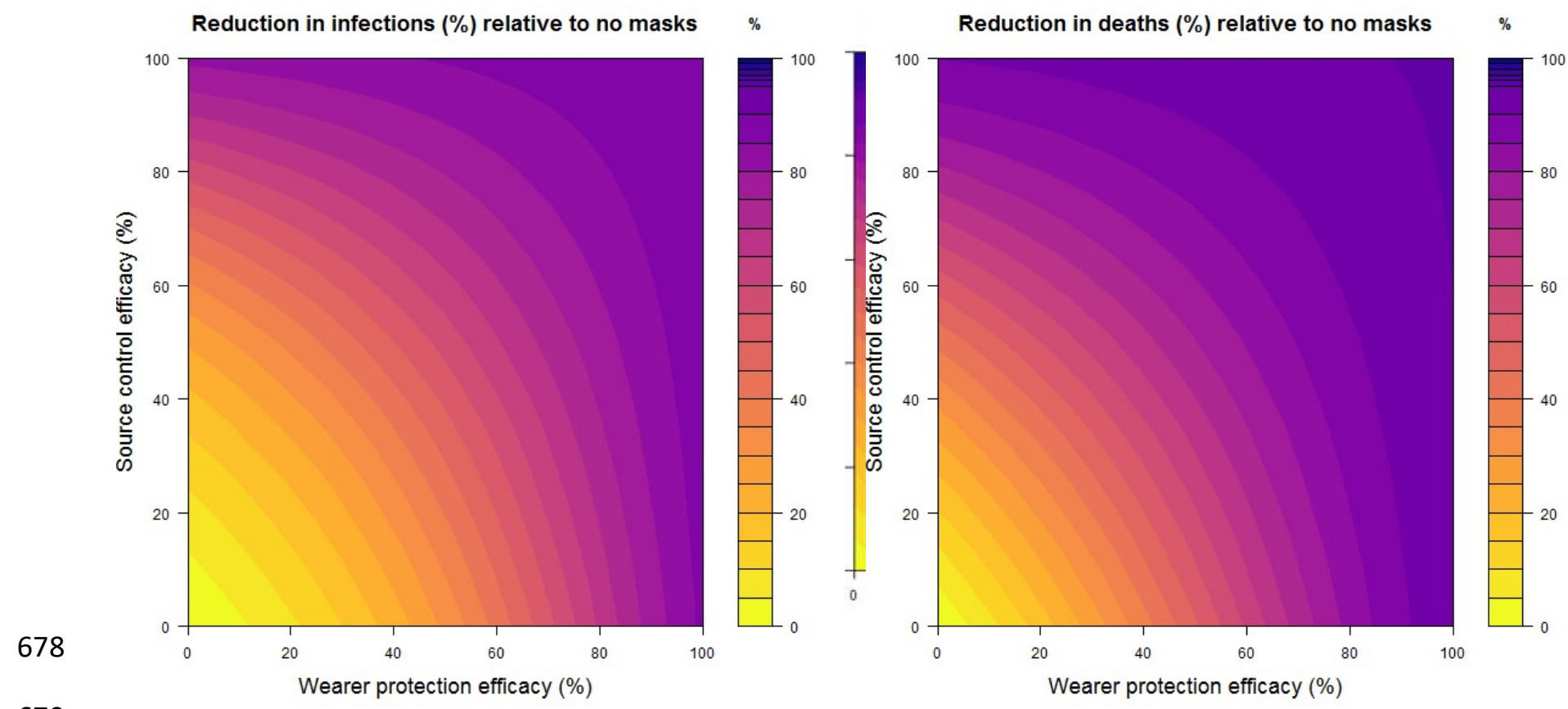

679

680

Figure 2

681 


\section{$684 \quad$ Figure 3}

685
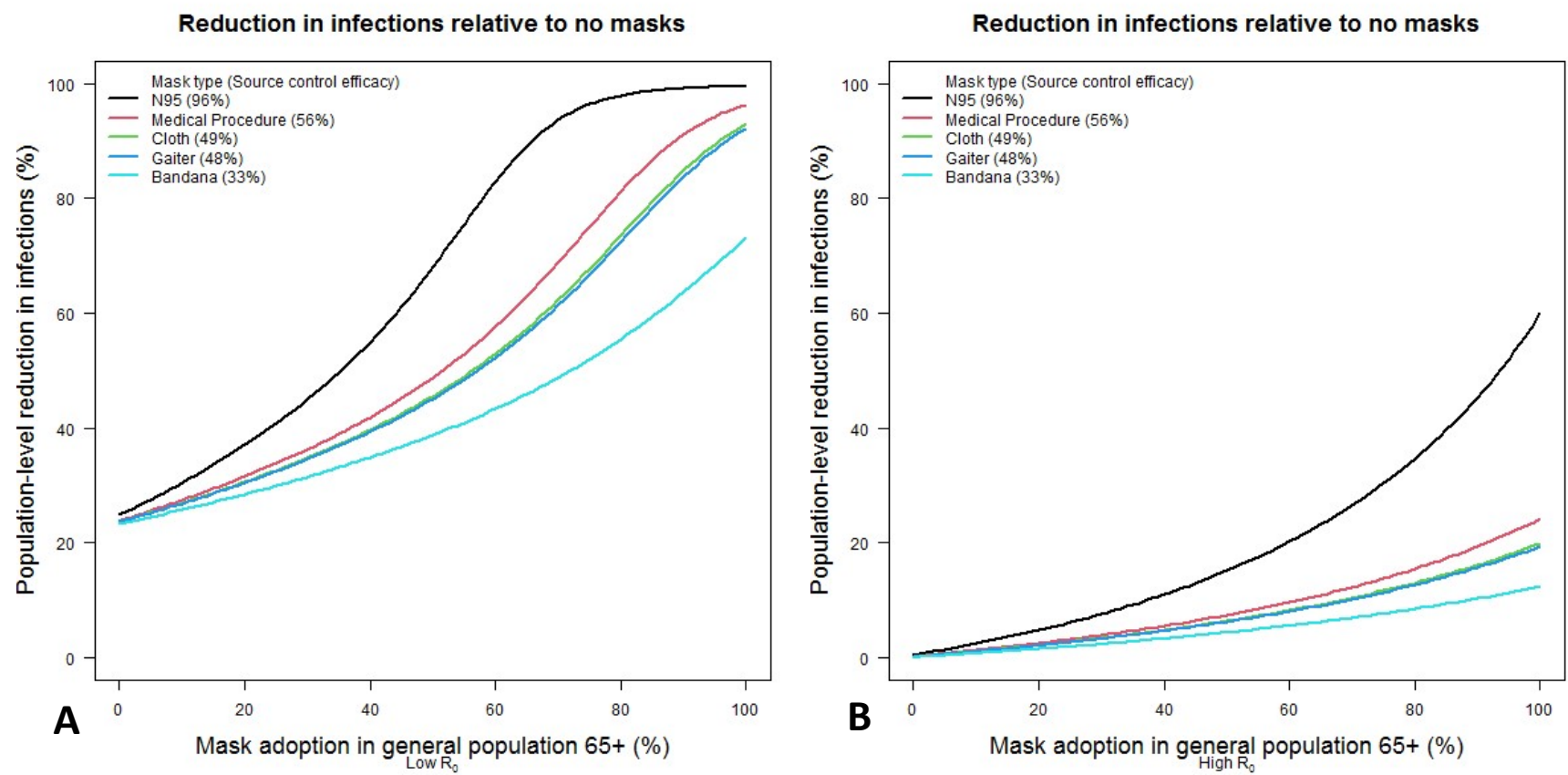
Effective reproduction number

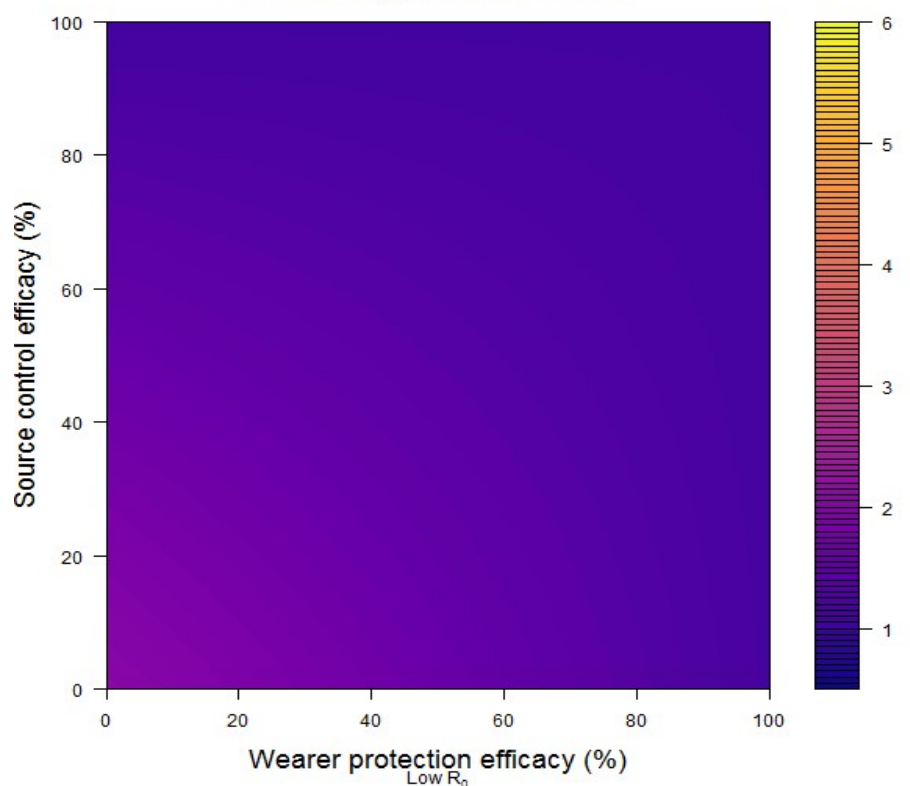

$R_{E}$
Effective reproduction number

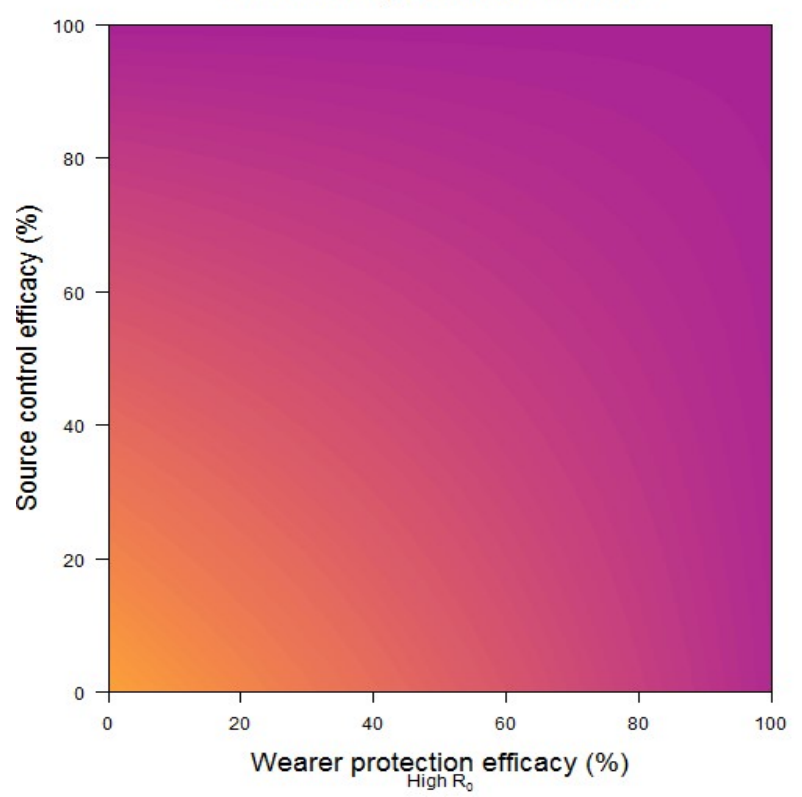

$R_{E}$

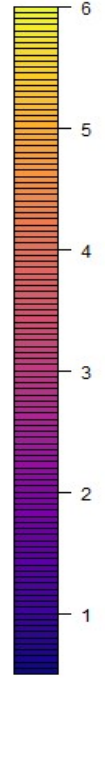

$687 \quad$ Figure 4 